\section{PPKC $\alpha$ mediated-HIF-1 $\alpha$ activation related to the morphological modifications occurring in neonatal myocardial tissue in response to severe and mild hyperoxia}

\author{
S. Zara, ${ }^{1}$ V. Macchi, ${ }^{2}$ R. De Caro, ${ }^{2}$ \\ M. Rapino, ${ }^{3}$ A. Cataldi, ${ }^{4}$ A. Porzionato ${ }^{2}$ \\ 'Department of Drug Sciences, University \\ G. D'Annunzio Chieti-Pescara, Chieti; \\ 2Department of Human Anatomy and \\ Physiology, University of Padua; \\ ${ }^{3}$ Institute of Molecular Genetics CNR, \\ Unit of Chieti; \\ ${ }^{4}$ Department of Medicine and Ageing \\ Sciences, University G. D'Annunzio \\ Chieti-Pescara, Chieti, Italy
}

\section{Abstract}

In premature babies birth an high oxygen level exposure can occur and newborn hyperoxia exposure can be associated with free radical oxygen release with impairment of myocardial function, while in adult animal models short exposure to hyperoxia seems to protect heart against ischemic injury. Thus, the mechanisms and consequences which take place after hyperoxia exposure are different and related to animals age. The aim of our work has been to analyze the role played by HIF-1 $\alpha$ in the occurrence of the morphological modifications upon hyperoxia exposure in neonatal rat heart. Hyperoxia exposure induces connective compartment increase which seems to allow enhanced blood vessels growth. An increased hypoxia inducible factor- $1 \alpha$ (HIF-1 $\alpha$ ) translocation and vascular endothelial growth factor (VEGF) expression has been found upon 95\% oxygen exposure to induce morphological modifications. Upstream pPKC- $\alpha$ expression increase in newborn rats exposed to $95 \%$ oxygen can suggest PKC involvement in HIF-1 $\alpha$ activation. Since nitric oxide synthase (NOS) are involved in heart vascular regulation, endothelial NOS (e-NOS) and inducible NOS (i-NOS) expression has been investigated: a lower eNOS and an higher iNOS expression has been found in newborn rats exposed to 95\% oxygen related to the evidence that hyperoxia provokes a systemic vasoconstriction and to the iNOS pro-apoptotic action, respectively. The occurrence of apoptotic events, evaluated by TUNEL and Bax expression analyses, seems more evident in sample exposed to severe hyperoxia. All in all such results suggest that in newborn rats hyperoxia can trigger oxygen free radical mediated membrane injury through a pPKC $\alpha$ mediated HIF-1 $\alpha$ signalling system, even though specificity of such response could be obtained by in vivo administration to the rats of specific inhibitors of PKC $\alpha$. This intracellular signalling can switch molecular events leading to blood vessels development in parallel to pro-apoptotic events due to an immature antioxidant defensive system in newborn rat hearts.

\section{Introduction}

In intensive care medicine, newborns are quite frequently exposed to high levels of oxygen for several hours or even days, for instance in extracorporeal membrane oxygenation or due to prematurity. ${ }^{1,2}$ It is known that premature newborns have decreased antioxidant defenses ${ }^{3,4}$ and severe myocardial dysfunctions have been reported in children exposed to extracorporeal membrane oxygenation. ${ }^{5}$ However, apart from its preconditioning and protecting effects on myocardial ischaemia/ infarction, the effects of hyperoxia exposure on heart have rarely been studied in the literature. $^{6-8}$ Exposure of seven-week-old rats to $100 \%$ oxygen for $60 \mathrm{~h}$ has not been observed to cause significant increase in lipid peroxidation in the heart, but in these rat hearts phospholipase C isozymes $\gamma 1$ and $\delta 1$ were increased and decreased, in the cytosolic and particulate fractions, respectively. ${ }^{6}$ From a functional point of view, $5 \mathrm{~h}$ of hyperoxia exposure $\left(\mathrm{PaO}_{2}=422 \mathrm{mmHg}\right)$ have been reported to cause significant reductions in contractility, systolic and mean arterial blood pressures, and significant increase in heart rate, in Yorkshire piglets. Moreover, in the left ventricle, the indicators of oxygen free radical-mediated membrane injury, malondialdehyde and 4-hydroxynonenal, were significantly elevated, in the presence of significant reduction of the superoxide dismutase and glutathione peroxidase activities. ${ }^{7}$ Conversely, exposure of adult mice to $40 \%$ oxygen for 10 days has been found to increase the concentrations of the antioxidant vitamin $\mathrm{E}$ in the heart. ${ }^{8}$ Recent studies have also evaluated the persistence of cardiovascular alterations in adulthood after neonatal hyperoxia exposure. In particular, newborn rats kept in $80 \% \mathrm{O}_{2}$ air from the third to the tenth postnatal day have been found to show in adulthood increased systolic and diastolic blood pressure, increased maximal vasoconstriction and sensitivity to angiotensin II (males only), and impaired endotheliumdependant vasodilatation to carbachol. Angiotensin II has been also found to signifi-
Correspondence: Susi Zara, Section of Human Anatomy, Faculty of Pharmacy, University G. d'Annunzio Chieti-Pescara, via dei Vestini 31, 66100 Chieti, Italy.

Tel. +39.0871 .3554521 - Fax: +39.0871 .3554568 . E-mail: s.zara@unich.it

Key words: HIF-1 $\alpha$, pPKC $\alpha$, neonatal myocardial tissue, hyperoxia.

Acknowledgements: this work was supported by 60\% MIUR, year 2011, Prof. Cataldi.

Received for publication: 10 October 2011

Accepted for publication: 29 November 2011.

This work is licensed under a Creative Commons Attribution NonCommercial 3.0 License (CC BYNC 3.0).

(OCopyright S. Zara et al., 2012

Licensee PAGEPress, Italy

European Journal of Histochemistry 2012; 56:e2 doi:10.4081/ejh.2012.e2

cantly increase superoxide generation to higher levels in adult rats exposed to hyperoxia during neonatal life. ${ }^{9}$ Thus, there are few data in the literature regarding the specific effect of hyperoxia in the first postnatal period and the mechanisms which take place after hyperoxia exposure are contradictory and not well known.

Since a role has been already assigned by our group to protein kinase C (PKC) isoforms in the regulation of rat neonatal heart development and growth ${ }^{10,11}$ and rat heart response to hypoxia exposure,,${ }^{12,13}$ our attention has been focused on the role played by PKC isoforms in the response to hyperoxia exposure in rat neonatal heart. PKC is a multigenic family of protein kinases whose activation is associated with the regulation of intracellular signalling molecules. ${ }^{14}$ In fact, PKC transfer the phosphate group of ATP or GTP to the hydroxyl group of a substrate protein, inducing its activation. ${ }^{15}$ Moreover, $\mathrm{O}_{2}$ concentration modifications imply chemical steps which transduce the signal inside the nucleus, leading to induction or repression of transcription. Among the molecules involved in this signal transduction pathway, hypoxia inducible factor-1 $\alpha$ (HIF- $1 \alpha$ ) is included. ${ }^{12,16} \mathrm{HIF}-1 \alpha$ has been originally identified by its binding to an hypoxia response element (HRE) required for transcriptional activation in response to reduced cellular $\mathrm{O}_{2}$ concentration, ${ }^{17}$ even if recent studies $^{18}$ have demonstrated the HIF-1 $\alpha$ involvement not only in the response to hypoxia but also to hyperoxia exposure. Moreover, HIF-1 $\alpha$ regulates several biological processes which counterbalance oxygen level variations by stimulating gene expression changes which 
lead to blood vessel growth, VEGF activation and consequently the following steps of angiogenesis. ${ }^{18,19}$

Thus, this work aimed at analyzing the role played by pPKC $\alpha$ mediated-HIF-1 $\alpha$ activation in the occurrence of the morphological modifications involved in oxygen homeostasis maintenance and in blood vessels organization in rat hearts exposed to different levels of hyperoxia in the first two postnatal weeks and in rats subjected to a following recovery period of normoxia, as elsewhere reported in another experimental model. ${ }^{20}$ In addition, since NOS enzymes, responsible of NO production, are involved in the vascular regulation during cardiac muscle development, ${ }^{21,22}$ e-NOS and i-NOS expression, constitutive and inducible molecule respectively, has been investigated along with apoptotic event occurrence.

\section{Materials and Methods}

\section{Animals and experimental procedure of hyperoxia exposure}

Female Sprague-Dawley rats (Harlan, Udine, Italy) and their offspring have been housed and handled in accordance with the guidelines of Helsinki Declaration and Local Ethical Committee. The study has been conducted on male or female rat pups kept together with their nursing mother. Mothers and litters have been placed in clear polished acrylic chambers provided with software enabling a continuous monitoring of $\mathrm{O}_{2}$ and $\mathrm{CO}_{2}$

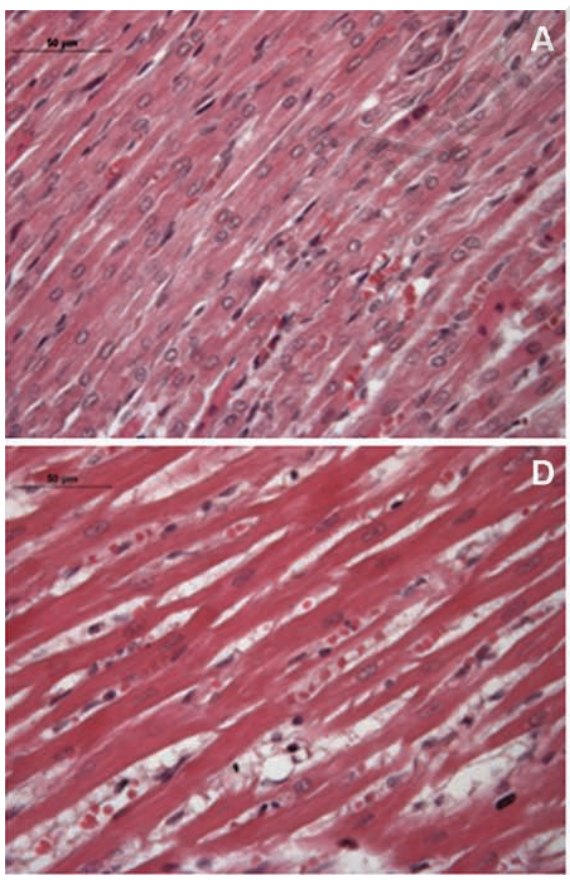

(BioSpherix, 0xyCycler model A84X0V, Redfield, NY, USA). Animals have been maintained under standardized conditions of light (12:12 h light-dark cycle, illumination onset 07.00 a.m.) at $24^{\circ} \mathrm{C}$. After term gestation, the newborn rats have been randomly distributed between the following experimental groups: a) newborn rats $(\mathrm{n}=10)$ raised in ambient air for 2 weeks; b) newborn rats ( $\mathrm{n}=10)$ exposed to $60 \%$ oxygen for 2 weeks; c) newborn rats $(n=10)$ exposed to $95 \%$ oxygen for 2 weeks; d) newborn rats $(\mathrm{n}=10)$ raised in ambient air for 6 weeks; e) newborn rats $(\mathrm{n}=10)$ exposed to $60 \%$ oxygen for 2 weeks and then kept in ambient air for further 4 weeks. The nursing dams have been rotated every one day to prevent any negative effects of hyperoxia on nursing.

At the experimental endpoints ( 2 weeks for groups a, b and c; 6 weeks for groups $d$ and e) animals have been sacrificed with Nembutal (40 mg/kg). Left ventricles have been excised from each rat, washed in phosphate-buffered saline (PBS) and cut into two blocks which have been fixed in 10\% phosphate-buffered formalin or frozen at $-80^{\circ} \mathrm{C}$.

\section{Light microscopy analysis and immunohistochemistry}

Tissues have been fixed in 10\% phosphatebuffered formalin for $72 \mathrm{~h}$ and dehydrated through ascending alcohols and xylene and then paraffin embedded. Samples have been then de-waxed (xylene and alcohol progressively lower concentrations) and the tissue sections, $5 \mu \mathrm{m}$ thick, have been processed for haematoxylin-eosin staining and for immuno-

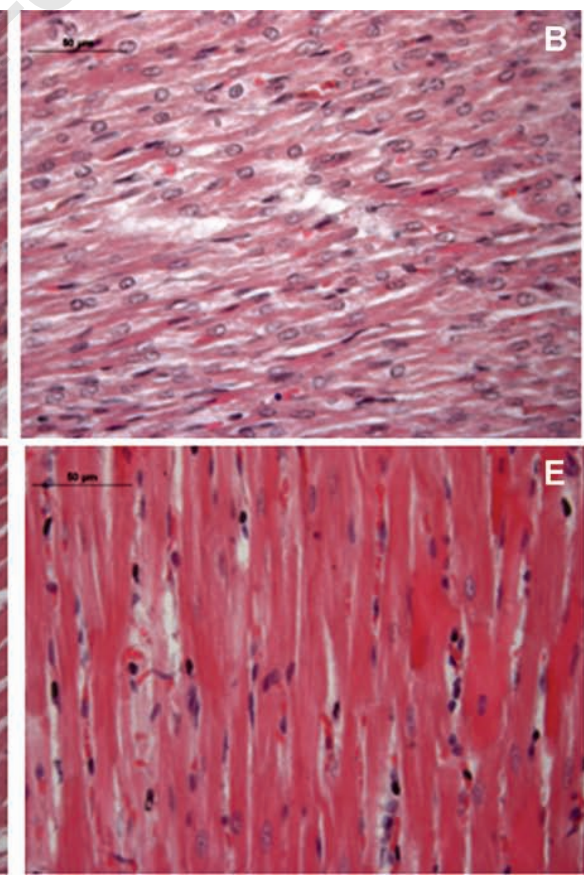

histochemical analysis.

In order to detect VEGF, HIF-1 $\alpha$ and Bax proteins, immunohistochemistry has been performed on $5 \mu \mathrm{m}$ thick sections by means of Ultravision LP Detection System HRP Polymer \& DAB Plus Chromogen (Lab Vision Thermo, CA, USA) (primary antibodies dilution 1:100). Tissue sections have been incubated in the presence of rabbit anti-VEGF, mouse anti-HIF$1 \alpha$ and anti-Bax antibodies (Santa Cruz Biotechnology, Santa Cruz, CA, USA). Sections have been incubated in the presence of specific HRP-conjugated secondary antibodies. Peroxidase has been developed using diaminobenzidin chromogen (DAB) and nuclei have been hematoxylin counterstained Negative controls have been performed by omitting the primary antibody. Samples have been then observed by means of Leica DM 4000 light microscopy (Leica Cambridge Ltd., Cambridge, UK) equipped with a Leica DFC 320 camera (Leica Cambridge Ltd.) for computerized images.

\section{Terminal-deoxinucleotidyl- transferase-mediated dUTP nick end-labeling analysis}

Terminal-deoxinucleotidyl-transferasemediated dUTP nick end-labeling (TUNEL) is a method of choice for a rapid identification and quantification of apoptotic cells. DNA strand breaks, yielded during apoptosis, can be identified by labeling free 3'-OH termini with modified nucleotides in an enzymatic reaction. Paraffin embedded tissue sections have been de-waxed and rehydrated. All steps have been

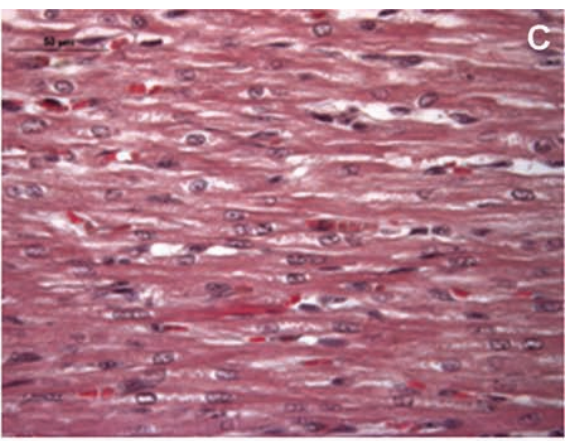

Figure 1. Hematoxylin-eosin staining of neonatal rat heart; A) ambient air; B) 60\% hyperoxia; C) 95\% hyperoxia; D) ambient air 6 weeks; E) $60 \%$ hyperoxia and ambient air 4 weeks. Scale bar 50 um. 
realized with FragEL DNA fragmentation Detection Kit according to the manufacturer's instructions (Calbiochem Merck, Cambridge, MA, USA). After two rinses in PBS, tissue slides have been dehydrated, mounted by using a permanent media and analyzed by light microscope (Leica Cambridge Ltd.). Five slides from each sample have been assessed, apoptotic cells count has been performed on ten fields per slide. Negative control has been performed by omitting the incubation in the presence of the enzymatic mixture, positive control (not shown) has been performed by treating one slide with DNAse I.

\section{Computerized morphometry measurements and image analysis}

After digitizing the images deriving from haematoxylin-eosin stained sections, Qwin Plus 3.5 software (Leica Cambridge Ltd.) overlay tools have been used to measure fiber diameter, muscle and connective compartment areas. Ten optical fields have been observed at $40 \times$ magnification and fiber diameter has been measured at nuclear level which is the widest portion of the fiber. Connective compartment area has been measured through the quantification of thresholded area for white/light pink color per ten fields of light microscope observation; muscle compartment area has been obtained by difference. Values have been logged on Microsoft Excel and processed for media, percentages and standard deviations.

After digitizing the images deriving from immunohistochemical stained sections, QWin Plus 3.5 software has been used to evaluate VEGF, HIF- $1 \alpha$ and Bax expression. Image analysis of protein expression has been performed through the quantification of thresholded area for immunohistochemical brown color per ten fields of light microscope observation.

QWin Plus 3.5 assessments have been logged to Microsoft Excel and processed for Standard Deviations and Histograms. The statistical significance of the results has been evaluated using the $t$-test and the Linear Regression Test, with $\mathrm{P}<0.05$.

\section{Western blotting analysis}

Heart lysates $(20 \mu \mathrm{g})$ have been electrophoresed and transferred to nitrocellulose membrane. Nitrocellulose membranes, blocked in 5\% non-fat milk, $10 \mathrm{mmol} / \mathrm{L}$ Tris $\mathrm{pH} 7.5,100$ $\mathrm{mmol} / \mathrm{L} \mathrm{NaCl}, 0.1 \%$ Tween-20, have been probed with mouse anti $\alpha$-actin, mouse anti $\beta$ tubulin antibodies (Sigma, (primary antibodies dilution 1:1000) mouse anti-collagen III, rabbit anti-VEGF, anti-iNOS and anti- eNOS and goat anti-p-PKC $\alpha$ polyclonal antibodies (Santa Cruz Biotechnology) (primary antibodies dilution 1:200) and then incubated in the presence of specific enzyme conjugated IgG horseradish peroxidase. Immunoreactive bands have been detected by ECL detection system (Amersham Int., Buckinghamshire, UK) and analysed by densitometry. Densitometric values, expressed as Integrated Optical Intensity (IOI), have been estimated in a CHEMIDOC XRS system by the QuantiOne 1-D analysis software (BIORAD, Richmond, CA, USA). Values obtained have been normalized basing on densitometric values of internal $\beta$ tubulin. Statistical analysis has been performed using the analysis of variance (ANOVA). Results have been expressed as mean \pm SD. Values of $\mathrm{P}<0.05$ have been considered statistically significant.

\section{Results}

Morphological features have been analyzed by hematoxylin-eosin staining and western blot analysis of connective and muscle compartments proteins. In newborn rat hearts raised in ambient air for 2 weeks organized myocardial cells, disclosing homogeneous diameter, are evidenced with in parallel blood vessels arranged. In newborn rat hearts exposed to mild hyperoxia ( $60 \%$ oxygen) for 2 weeks less organized cells with different diameters are distinguishable, connective compartment is increased respect to two weeks old rat heart and blood vessels dilata-

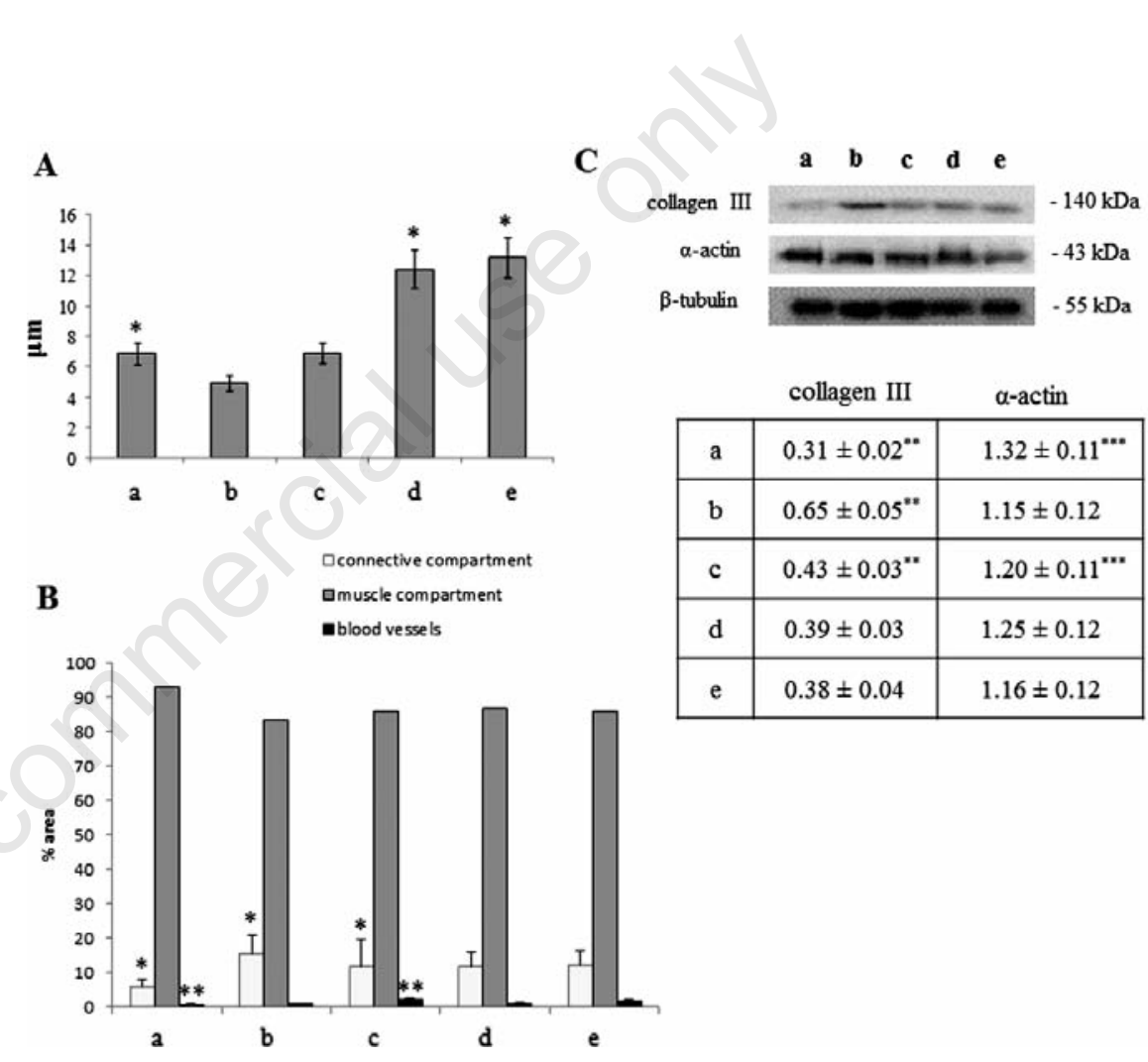

Figure 2. Morphometric analysis of neonatal rat heart performed on hematoxylin-eosin stained slides; a) ambient air; b) 60\% hyperoxia; c) 95\% hyperoxia; d) ambient air 6 weeks; e) $60 \%$ hyperoxia and ambient air 4 weeks. A) Fiber diameter measurements expressed as mean values $( \pm \mathrm{SD})$ assessed by direct visual measurement of ten muscle fibers for each of three slides per each of 5 samples at 40x magnification; *ambient air 6 weeks vs ambient air $\mathrm{P}<0.05 ;{ }^{*} \mathbf{6 0} \%$ hyperoxia and ambient air 4 weeks $v$ s ambient air $\mathrm{P}<0.05$. B) Blood vessels, connective and muscle compartment measurements expressed as $\%$ area mean $( \pm S D)$ assessed by direct visual counting of ten fields for each of three slides per each of 5 samples; ${ }^{*} 60 \%$ hyperoxia connective compartment $\%$ area $v s$ ambient air connective compartment \% area $\mathrm{P}<\mathbf{0 . 0 5}$; ${ }^{*} \mathbf{9 5 \%}$ hyperoxia connective compartment vs ambient air connective compartment $\%$ area $\mathrm{P}<0.05$; ${ }^{* *} 95 \%$ hyperoxia blood vessels $\%$ area $v$ ambient air blood vessels \% area $\mathrm{P}<\mathbf{0 . 0 5}$. C) Western blotting analysis of collagen III and $\alpha-$ actin expression; each membrane has been probed with anti $\beta$-tubulin antibody to verify loading evenness. The most representative out of three separate experiments is shown. Data are the densitometric measurements of protein bands expressed as Integrated Optical Intensity (IOI) mean $( \pm \mathrm{SD})$ of three separate experiments; ${ }^{* *} 60 \%$ hyperoxia collagen III $v s$ ambient air collagen III $\mathrm{P}<0.05 ;{ }^{* *} \mathbf{9 5} \%$ hyperoxia collagen III $v \boldsymbol{s}$ ambient air collagen III $\mathrm{P}<0.05 ;{ }^{* *} 60 \%$ hyperoxia collagen III $v s \quad 95 \%$ hyperoxia collagen III; ${ }^{* *} 95 \%$ hyperoxia $\alpha$-actin $v$ ambient air $\alpha$-actin $\mathrm{P}<0.05$. 
tion can be observed. Newborn rats exposed to severe hyperoxia ( $95 \%$ oxygen) for 2 weeks showed a mortality rate of about $30 \%$. However, their hearts appear less suffering than $60 \%$ oxygen exposed ones, disclosing myocardial cells well arranged even if an increased connective compartment, when compared to hyperoxia unexposed sample, is recognizable.

Newborn rat hearts raised in ambient air for 6 weeks and newborn rat hearts exposed to $60 \%$ oxygen for 2 weeks and then kept in ambient air for other 4 weeks do not disclose any significant morphological differences, while a physiological increase, due to the growth of the diameter of the cell, which reaches the definitive elongated shape occurs (Figures 1 and 2A). Western blot analysis for Collagen III, a physiological protein of connective tissue, has been performed confirming an increase of connective tissue along with blood vessels growth in newborn rat hearts exposed to $60 \%$ and $95 \%$ oxygen compared to newborn rat hearts raised in ambient air for 2 weeks. In parallel, the expression of a specific myocardial tissue protein, $\alpha$-actin, has been checked, showing an higher expression in hyperoxia unexposed samples when compared to exposed ones (Figure 1 and Figure 2 B,C).

Since morphological modifications are induced by molecular events, we have investigated the expression of molecules involved in oxygen homeostasis maintenance. As HIF-1 $\alpha$ plays a pivotal role in the cellular signalling after hypoxia/hyperoxia exposure ${ }^{18}$ and its heterodimerization with Ahr nuclear translocator (ARNT) happens into the nucleus and is required for DNA binding and transactivation, ${ }^{23}$ we have evaluated its possible translocation at nuclear level by immunohistochemical analysis. A significant increase of HIF- $1 \alpha$ positive nuclei percentage in newborn rat hearts exposed to $95 \%$ oxygen for 2 weeks is evidenced, when compared to 2 weeks $60 \%$ oxygen exposed ones (Figure 3). Newborn rat hearts raised in ambient air for 6 weeks showed lower percentage of HIF-1 $\alpha$ positive nuclei than newborn rat hearts raised in ambient air for 2 weeks. Conversely, newborn rat hearts exposed to $60 \%$ oxygen for 2 weeks and then kept in ambient air for other 4 weeks show higher percentage of nuclear positivity than newborn rat hearts raised in ambient air for 2 weeks and newborn rat hearts exposed to $60 \%$ oxygen for 2 weeks.

HIF-1 $\alpha$ activation, in turn, can induce blood vessels growth factors modifications and, as a consequence, an altered blood vessels organization. ${ }^{19}$ Thus, VEGF expression has been evaluated by immunohistochemical and western blot analyses. A higher level of VEGF expression in newborn rat hearts exposed to $95 \%$ oxygen for 2 weeks than in the other experimental conditions is disclosed (Figure 4). These results have lead us to focus attention on molecules which could be involved in HIF-1 $\alpha$ phosphorylation such as PKC. A higher expression for active PKC $\alpha$ (p-PKC $\alpha$ ) is found in newborn rat hearts exposed to $95 \%$ oxygen for 2 weeks than in the other specimens (Figure 5). As previous
A
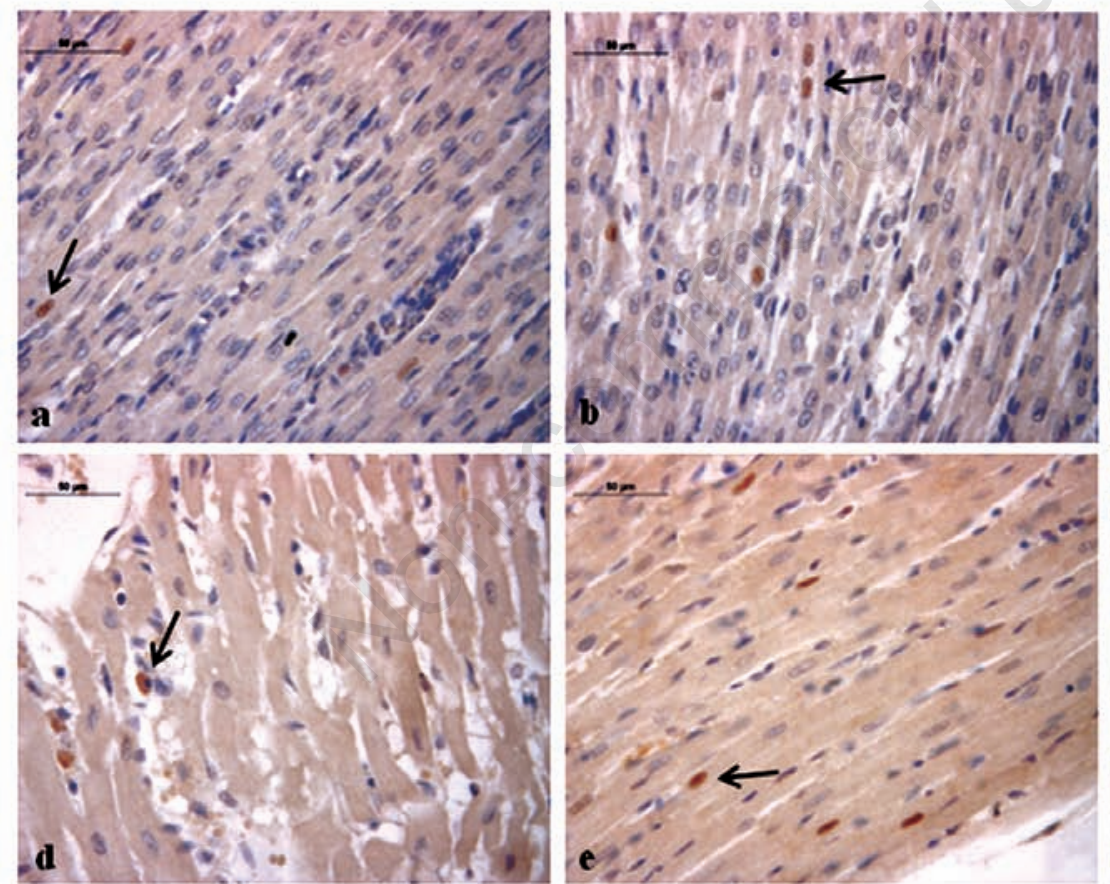
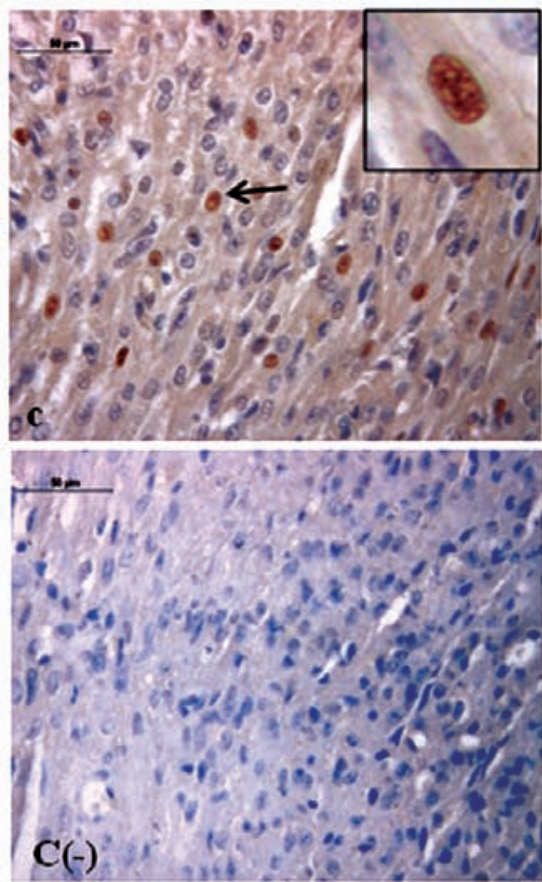

B

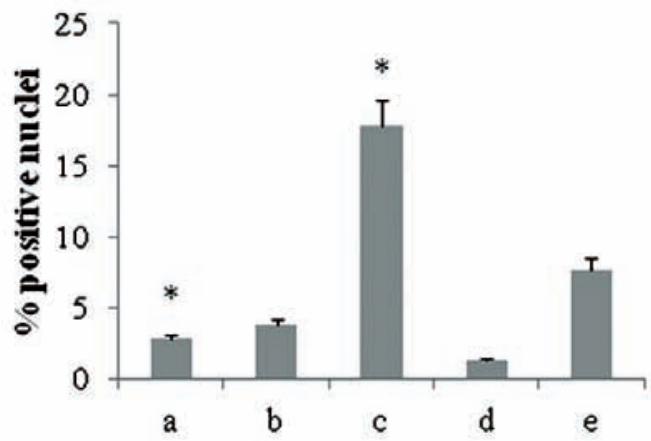

Figure 3. A) Immunohistochemical detection of HIF-1 $\alpha$ expression in neonatal rat heart; a) ambient air; b) $60 \%$ hyperoxia; c) 95\% hyperoxia; d) ambient air 6 weeks; e) $60 \%$ hyperoxia and ambient air 4 weeks; c(-) negative control; arrows indicate HIF$1 \alpha$ positive nuclei; scale bar $50 u \mathrm{~m}$; inset shows HIF-1 $\alpha$ nuclear staining. B) Graphic representation of HIF-1 $\alpha$ positive nuclei $\%$ $( \pm$ SD) densitometric analysis determined by direct visual counting of ten fields (mean values) for each of three slides per sample at 40x magnification; ${ }^{*} 95 \%$ hyperoxia $v$ s ambient air $\mathrm{P}<0.05$. 
studies have demonstrated NOS role in blood vessels regulation in cardiac muscle, endothelial (eNOS) and inducible (iNOS) nitric oxide synthase expression has been also investigated by western blot analysis evidencing a lower expression for eNOS molecule and an higher iNOS expression in newborn rat hearts exposed to $95 \%$ oxygen for 2 weeks than in other experimental conditions, suggesting the occurrence of an oxidative stress (Figure 6).

Therefore, by TUNEL analysis and Bax proapoptotic factor expression apoptotic events have been detected. Both TUNEL analysis and immunohistochemical analysis of Bax indicated within myocardial cells a higher positivity (Figures 7 and 8 , respectively) in newborn rat hearts exposed to $95 \%$ oxygen for 2 weeks.

\section{Discussion}

In newborn children requiring critical care, such as in extracorporeal membrane oxygenation, undergoing cardiopulmonary bypass, or in premature babies birth, an high oxygen level exposure (hyperoxia), for different time intervals, can occur., ${ }^{1,2}$ The rationale for the use of hyperoxia is to ensure adeguate oxygen delivery, even though the detrimental effects of such treatment has not always been considered. Moreover, previous studies have associated newborn hyperoxia exposure with strong free radical oxygen release which can induce a partial impairment of myocardial function due to newborn higher sensitivity to stresses. ${ }^{7}$ Other investigations on adult animal models have shown that short exposure

A

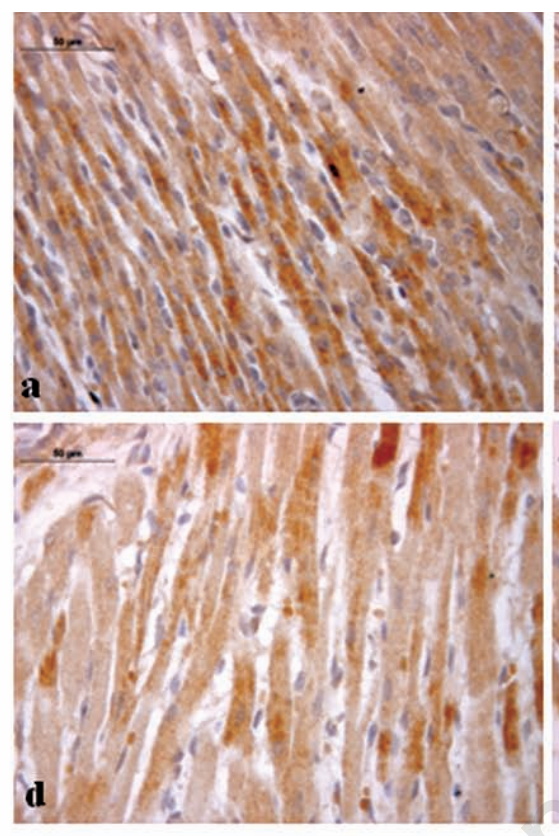

B

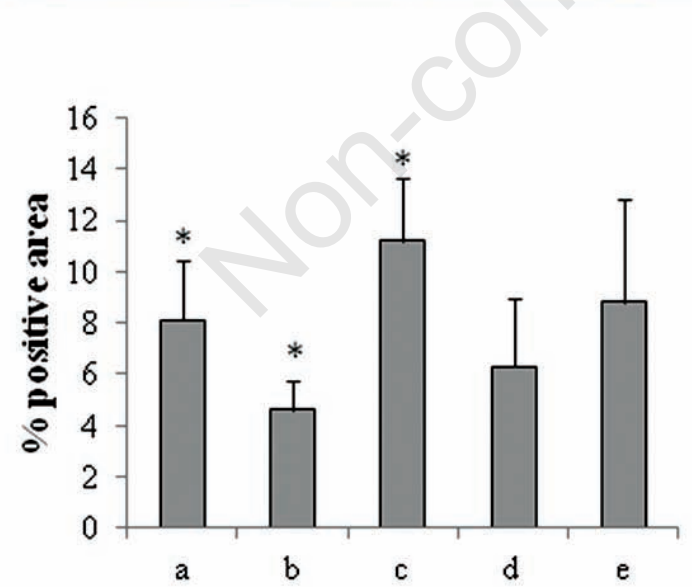

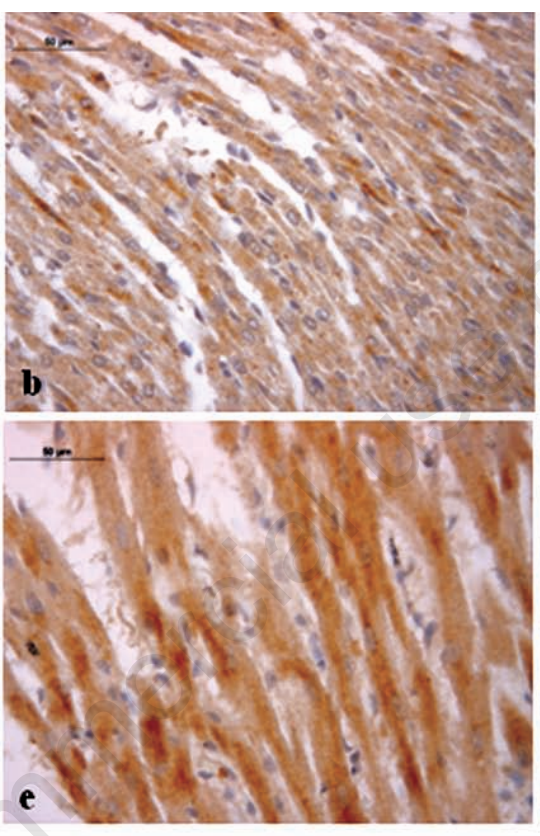

C

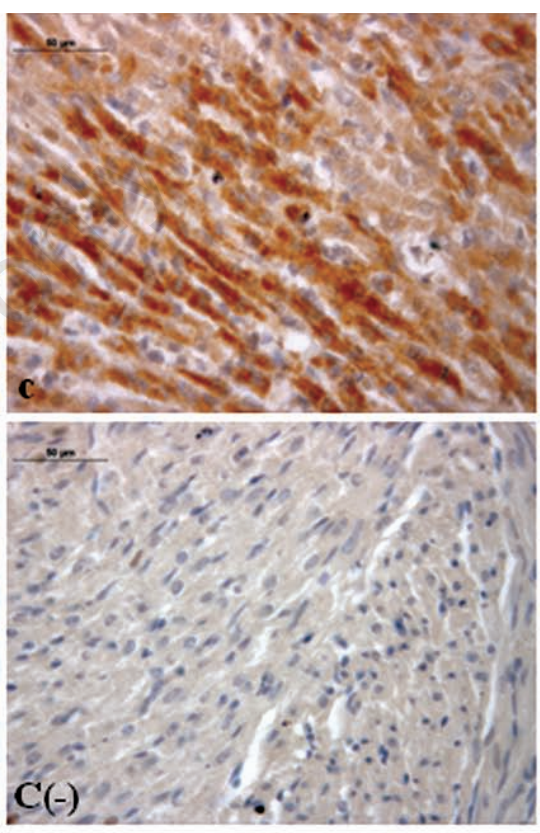

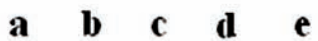

VEGF
$\beta$-tubulin
\begin{tabular}{|c|l|}
\hline a & $0.081 \pm 0.007^{* *}$ \\
\hline b & $0.062 \pm 0.006^{* *}$ \\
\hline c & $0.150 \pm 0.09^{* *}$ \\
\hline d & $0.071 \pm 0.008$ \\
\hline e & $0.082 \pm 0.007$ \\
\hline
\end{tabular}

Figure 4. A) Immunohistochemical detection of VEGF expression in neonatal rat heart; a) ambient air; b) $60 \%$ hyperoxia; c) $95 \%$ hyperoxia; d) ambient air 6 weeks; e) $60 \%$ hyperoxia and ambient air 4 weeks; c(-) negative control; scale bar $50 \mu \mathrm{m}$. B) Graphic representation of VEGF $\%$ positive area $( \pm$ SD) densitometric analysis determined by direct visual counting of ten fields (mean values) for each of three slides per sample at 40x magnification; ${ }^{* 60} \%$ hyperoxia $v$ s ambient air $\mathrm{P}<0.05 ;{ }^{*} 95 \%$ hyperoxia $v s$ ambient air $\mathrm{P}<0.05 ;{ }^{*} 95 \%$ hyperoxia vs $\mathbf{6 0 \%}$ hyperoxia $\mathrm{P}<0.05$. C) Western blotting analysis of VEGF expression; each membrane has been probed with anti $\beta$ tubulin antibody to verify loading evenness. The most representative out of three separate experiments is shown. Data are the densitometric measurements of protein bands expressed as Integrated Optical Intensity (IOI) mean $( \pm \mathrm{SD})$ of three separate experiments; ${ }^{* *} 95 \%$ hyperoxia vs ambient air $\mathrm{P}<0.05 ;{ }^{*} 95 \%$ hyperoxia vs $60 \%$ hyperoxia $\mathrm{P}<0.05$. 


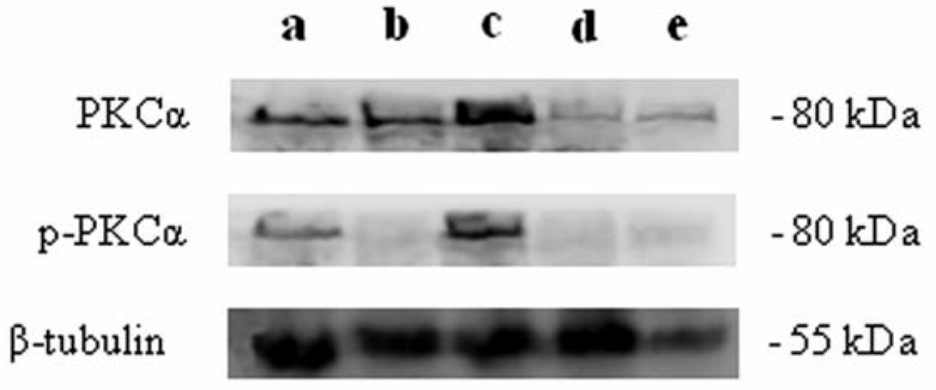

\begin{tabular}{|c|c|c|}
\hline & PKCo & p-PKCo \\
\hline a & $0.37 \pm 0.020^{*}$ & $0.30 \pm 0.020^{* *}:$ \\
\hline b & $0.34 \pm 0.024^{*}$ & $0.15 \pm 0.001$ \\
\hline c & $0.69 \pm 0.052^{*}$ & $0.68 \pm 0.040^{* *}$ \\
\hline d & $0.21 \pm 0.015$ & $0.17 \pm 0.010$ \\
\hline e & $0.20 \pm 0.017$ & $0.19 \pm 0.010$ \\
\hline
\end{tabular}

Figure 5. Western blotting analysis of $\mathrm{PKC} \alpha$ and $\mathrm{p}-\mathrm{PKC} \alpha$ expression in neonatal rat heart; a) ambient air; b) 60\% hyperoxia; c) $95 \%$ hyperoxia; d) ambient air 6 weeks; e) $60 \%$ hyperoxia and ambient air 4 weeks. Each membrane has been probed with anti $\beta$ tubulin antibody to verify loading evenness. The most representative out of three separate experiments is shown. Data are the densitometric measurements of protein bands expressed as Integrated Optical Intensity (IOI) mean $( \pm S D)$ of three separate experiments; ${ }^{*} 95 \%$ hyperoxia PKC $\alpha$ vs ambient air PKC $\alpha \quad \mathbf{P}<0.05 ;{ }^{*} \mathbf{6 0} \%$ hyperoxia PKC $\alpha$ vs ambient air PKC $\alpha$ $\mathbf{P}<0.05$; ${ }^{* *}$ 95\% hyperoxia $\mathrm{p}-\mathrm{PKC} \alpha$ vs ambient air $\mathrm{p}-\mathrm{PKC} \alpha \mathrm{P}<0.05$.

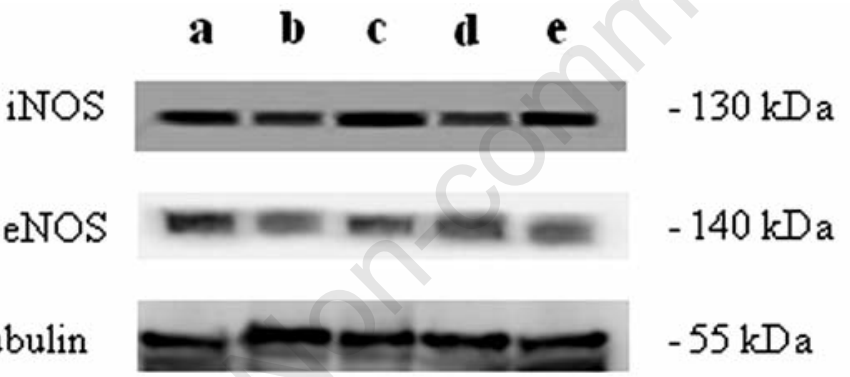

\begin{tabular}{|c|c|c|}
\hline & e-NOS & i-NOS \\
\hline a & $2.05 \pm 0.12 *$ & $0.036 \pm 0.002^{* *}$ \\
\hline b & $0.76 \pm 0.05$ & $0.026 \pm 0.001$ \\
\hline c & $1.06 \pm 0.09 *$ & $0.049 \pm 0.004^{* * \cdots}$ \\
\hline d & $0.47 \pm 0.04$ & $0.018 \pm 0.001$ \\
\hline e & $0.56 \pm 0.05$ & $0.030 \pm 0.002$ \\
\hline
\end{tabular}

Figure 6. Western blotting analysis of e-NOS and i-NOS expression in neonatal rat hearts; a) ambient air; b) $60 \%$ hyperoxia; c) $95 \%$ hyperoxia; d) ambient air 6 weeks; e) $60 \%$ hyperoxia and ambient air 4 weeks. Each membrane has been probed with anti $\beta$ tubulin antibody to verify loading evenness. The most representative out of three separate experiments is shown. Data are the densitometric measurements of protein bands expressed as Integrated Optical Intensity (IOI) mean $( \pm S D)$ of three separate experiments; ${ }^{*} 95 \%$ hyperoxia e-NOS vs ambient air e-NOS $\mathrm{P}<0.05$; ${ }^{* *} \mathbf{9 5 \%}$ hyperoxia i-NOS $v s$ ambient air i-NOS $\mathbf{P}<0.05$. 
to normobaric hyperoxia protects the isolated heart against ischemic injury and reperfusion-induced arrhythmias. ${ }^{24}$ Thus, the mechanisms and the following events which take place after hyperoxia exposure are different and probably related to the age of animals. The aim of our work was to analyze the role played by HIF-1 $\alpha$ in the occurrence of the morphological modifications upon $60 \%$ and 95\% oxygen exposure in neonatal rat heart.

Mild and severe hyperoxia exposure does not induce any fiber diameter modification but connective compartment increases along with enhanced blood vessels growth, as already demonstrated in different experimental models. ${ }^{18}$ These morphological modifications are induced by molecular events which activate the transcription of genes involved in oxygen homeostasis maintenance. Among these factors, HIF-1 $\alpha$, which expression undergoes strong modifications not only after hypoxia but also after hyperoxia exposure, ${ }^{18}$ plays a pivotal role. An increased HIF- $1 \alpha$ nuclear translocation is found upon $95 \%$ oxy- gen exposure, suggesting an evident HIF-1 $\alpha$ activation after severe hyperoxia condition exposure, according to Wikenheiser. In parallel, a strong VEGF increased expression in newborn rat hearts exposed to $95 \%$ oxygen for 2 weeks sample is found, supporting the hypothesis of HIF-1 $\alpha$-VEGF mediated activation. However little information is available about hyperoxic signal transduction from membrane receptor to nucleus. As PKC involvement in HIF-1 $\alpha$ phosphorylation/activation signalling during neonatal rat heart development and in response to intermittent hypoxia has been already demonstrated by our group, ${ }^{11,12}$ pPKC- $\alpha$ expression has been checked revealing an increased level in newborn rat hearts exposed to severe hyperoxia which suggests a possible role for this molecule in HIF-1 $\alpha$ activation upon hyperoxia exposure. The specificity of such response should be confirmed by in vivo administration of specific inhibitors of PKC $\alpha$ to the rats, although PKC $\alpha$ inhibitors toxic effect is well known ${ }^{25}$ and, in addition, PKC $\alpha$ inhibitor (bisindolylmaleimide VIII) has been used only in adult C57BL/6 mice not in newborns. ${ }^{26}$ Moreover, since NOS are involved not only in physiological and pathological events but also in the vascular regulation of cardiac muscle, ${ }^{21}$ e-NOS and i-NOS expression has been investigated. A lower expression of eNOS in newborn rat hearts exposed to $95 \%$ oxygen for 2 weeks, when compared to unexposed ones, could be related to the evidence that hyperoxia induces a systemic vasoconstriction, accordingly to different previous studies which have evidenced that hyperoxic peripheral vasoconstriction is linked to an altered endothelial function eliciting nitric oxide basal release reduction. ${ }^{27}$

At the same time, i-NOS increased expression in newborn exposed to $95 \%$ of hyperoxia probably justifies the pro-apoptotic action assigned to this molecule. ${ }^{28}$ This result is strongly confirmed by TUNEL and Bax expression analyses which evidence in newborn rat hearts exposed to $95 \%$ oxygen for 2 weeks an increased percentage of apoptotic cells which
A

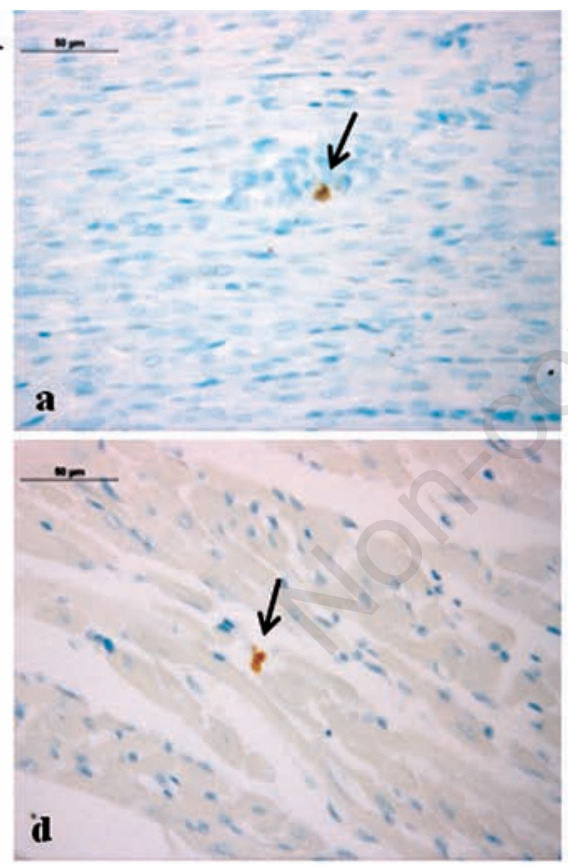

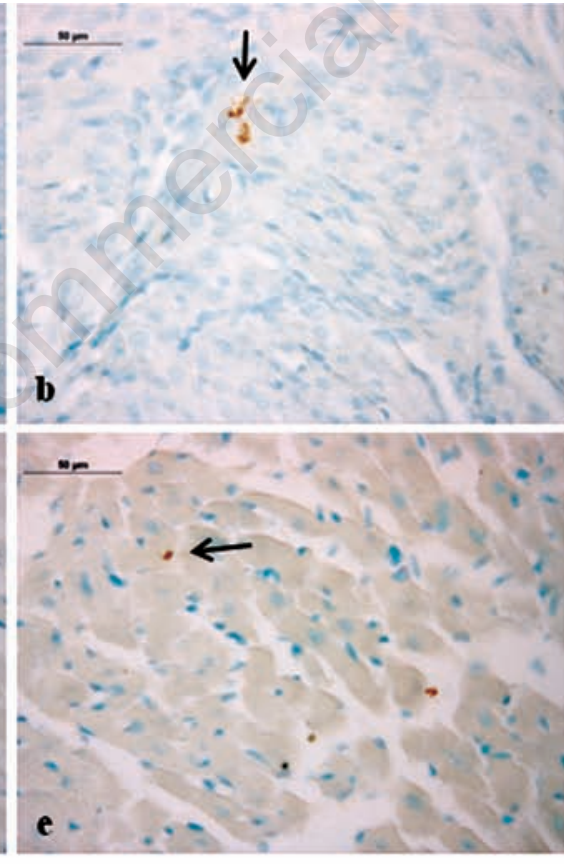

B

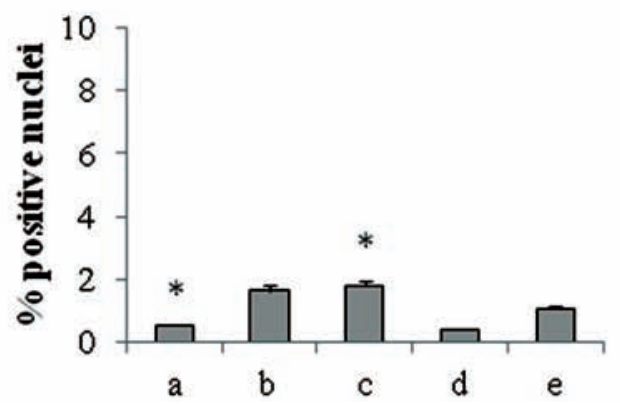

Figure 7. A) TUNEL detection of apoptotic nuclei in neonatal rat hearts; a) ambient air; b) $60 \%$ hyperoxia; c) $95 \%$ hyperoxia; d) ambient air 6 weeks; e) $60 \%$ hyperoxia and ambient air 4 weeks; c(-) negative control. Arrows indicate TUNEL positive nuclei (brown), negative nuclei are blue; scale bar $50 \mu \mathrm{m}$; B) Graphic representation of TUNEL positive nuclei $\%( \pm \mathrm{SD})$ densitometric analysis determined by direct visual counting of ten fields (mean values) for each of three slides per sample; ${ }^{*} \mathbf{9 5} \%$ hyperoxia $v$ s ambient air $\mathrm{P}<0.05$. 
A
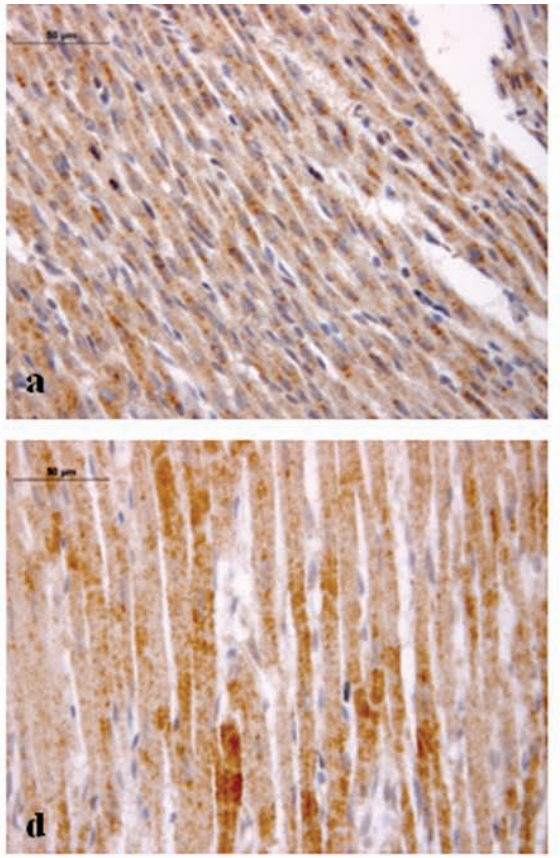
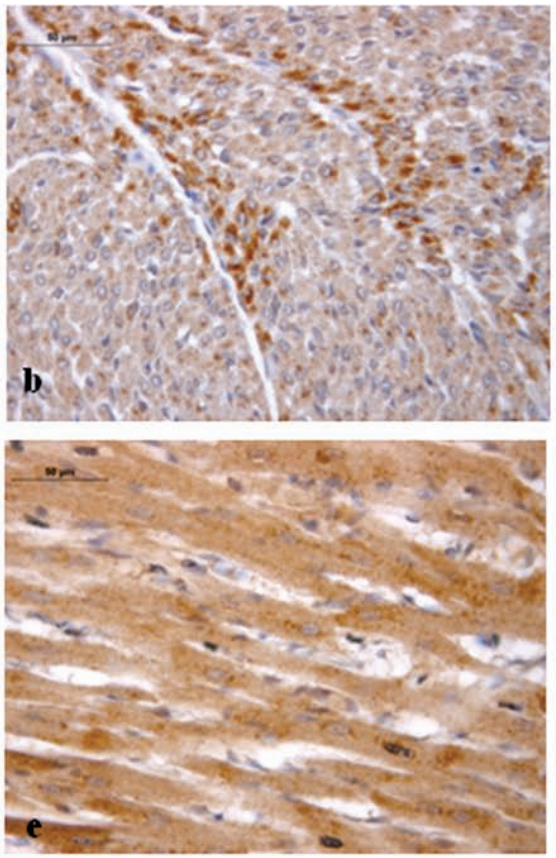
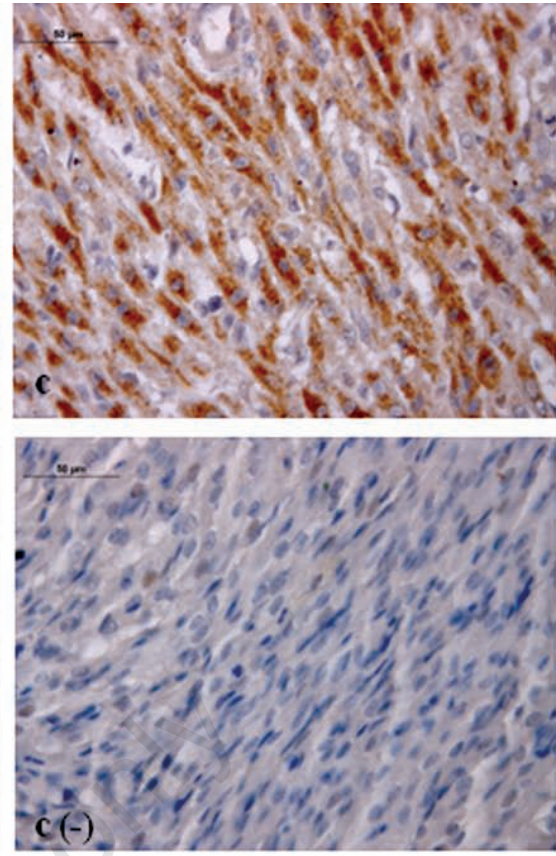

B

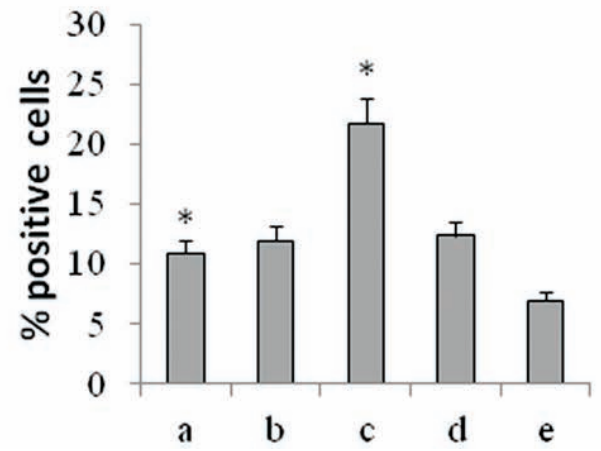

Figure 8. A) Immunohistochemical detection of Bax positive cells in different experimental points; magnification 40x; a) ambient air; b) $60 \%$ hyperoxia; c) $95 \%$ hyperoxia; d) ambient air 6 weeks; e) $60 \%$ hyperoxia and ambient air 4 weeks; $c(-)$ negative control; scale bar 50 $\mu \mathrm{m}$; B) Graphic representation of Bax positive cells $\%( \pm S D)$ determined by direct visual counting of ten fields (mean values) for each of three slides per sample; ${ }^{*} 95 \%$ hyperoxia $v s$ ambient air $\mathrm{P}<0.05$. can be due to the immature antioxidant defensive system of neonatal heart, as elsewhere reported. ${ }^{7}$ Newborn rat hearts exposed to $95 \%$ oxygen for 2 weeks seem to be more sensitive to hyperoxia injury, while, newborn rat hearts raised in ambient air for 6 weeks disclose a lower rate of apoptotic cells due to their ability to activate antioxidant defensive systems.

All the findings suggest that in newborn rats hyperoxia can trigger oxygen free radical mediated membrane injury through a pPKC $\alpha$ mediated HIF-1 $\alpha$ signalling system, even though specificity of such response could be obtained by in vivo administration of specific inhibitors of PKC $\alpha$ to the rats. This intracellular signalling can switch molecular events leading to blood vessels development in parallel to pro-apoptotic events due to an immature anti-oxidant defensive system in newborn rat hearts.

\section{References}

1. Wittnich C, Torrance SM, Carlyle CE. Effects of hyperoxia on neonatal myocardial energy status and response to global ischemia. Ann Thorac Surg 2000;70:212531.

2. Allen BS, Barth MJ, Ilbawi MN. Pediatric myocardial protection: an overview. Semin Thorac Cardiovasc Surg 2001;13:56-72.

3. Thibeault DW. The precarious antioxidant defenses of the preterm infant. Am J Perinatol 2000; 17:167-81.

4. Saugstad OD. Oxidative stress in the newborn-a 30-year perspective. Biol Neonate 2005;88:228-36.

5. Hirschl RB, Heiss KF, Bartlett RH. Severe myocardial dysfunction during extracorporeal membrane oxygenation. J Pediatr Surg 1992;27:48-53.

6. Nagasawa K, Tanino H, Shimohama S, Fujimoto S. Effects of hyperoxia and acrylonitrile on the phospholipase $\mathrm{C}$ isozyme protein levels in rat heart and brain. Life Sci 2003;73:1453-62.

7. Bandali KS, Belanger MP, Wittnich C. Hyperoxia causes oxygen free radicalmediate membrane injury and alters myocardial function and hemodynamics in the newborn. Am J Physiol Heart Circ Physiol 2004;287:553-9.

8. Lee ES, Smith WE, Quach HT, Jones BD, Santilli SM, Vatassery GT. Moderate hyperoxia (40\%) increases antioxidant levels in mouse tissue. J Surg Res 2005;127:80-4.

9. Yzydorczyk C, Comte B, Cambonie G, Lavoie JC, Germain N, Ting Shun Y, et al. Neonatal oxygen exposure in rats leads to cardiovascular and renal alterations in adulthood. Hypertension 2008;52:889-95.

10. Centurione L, Di Giulio C, Santavenere E, Cacchio M, Sabatini N, Rapino C, et al. Protein kinase $\mathrm{C}$ zeta regulation of hypertrophic and apoptotic events occurring during rat neonatal heart development and growth. Int J Immunopathol Pharmacol 2005;18:49-58 
11. Zara S, Bosco D, Di Giulio C, Antonucci A, Cataldi A. Protein kinase C alpha early activates splicing factor SC-35 during postnatal rat heart development. J Biol Regul Homeost Agents 2009;23:45-54.

12. Cataldi A, Bianchi G, Rapino C, Sabatini N, Centurione L, Di Giulio C, et al. Molecular and morphological modifications occurring in rat heart exposed to intermittent hypoxia: role for protein kinase $\mathrm{C}$ alpha. Exp Gerontol 2004;39:395-405.

13. Cataldi A, Zingariello M, Rapino M, Zara S, Daniele F, Di Giulio C, et al. Effect of hypoxia and aging on PKC delta-mediated SC-35 phosphorylation in rat myocardial tissue. Anat Rec 2009;292:1135-42.

14. Battaini F, Pascale. A Protein kinase C signal transduction regulation in physiological and pathological aging. Ann N Y Acad Sci 2005;1057:177-92.

15. Sridhar R, Hanson-Painton 0, Cooper DR. Protein kinases as therapeutic targets. Pharm Res 2000;17:1345-53.

16. Koshikawa N, Hayashi J, Nakagawara A, Takenaga K. Reactive oxygen species-generating mitochondrial DNA mutation upregulates hypoxia-inducible factor-1alpha gene transcription via phosphatidylinositol 3-kinase-Akt/protein kinase C/histone deacetylase pathway. J Biol Chem 2009; 284:33185-94.
17. Semenza GL. Oxygen homeostasis. Wiley Interdiscip Rev Syst Biol Med 2010;2:33661.

18. Wikenheiser J, Wolfram JA, Gargesha M, Yang K, Karunamuni G, Wilson DL, et al. Altered hypoxia-inducible factor-1 alpha expression levels correlate with coronary vessel anomalies. Dev Dyn 2009;238:2688700 .

19. Zakrzewicz A, Secomb TW, Pries AR. Angioadaptation: keeping the vascular system in shape. News Physiol Sci 2002;17: 197-201.

20. Park MS, Sohn MH, Kim KE, Park MS, Namgung R, Lee C. 5-Lipoxygenase-activating protein (FLAP) inhibitor MK-0591 prevents aberrant alveolarization in newborn mice exposed to $85 \%$ oxygen in a dose- and time-dependent manner. Lung 2011;189:43-50.

21. Karimi G, Fatehi Z, Gholamnejad Z. The role of nitric oxide and protein kinase $\mathrm{C}$ in lipopolysaccharidemediated vascular hyporeactivity. J Pharm Pharm Sci 2006;9: 119-23.

22. Mujoo K, Sharin VG, Bryan NS, Krumenacker JS, Sloan C, Parveen S, et al. Role of nitric oxide signaling components in differentiation of embryonic stem cells into myocardial cells. Proc Natl Acad Sci U S A 2008;105:18924-9.
23. Hoffman EC, Reyes H, Chu FF, Sander F, Conley LH, Brooks BA, et al. Cloning of a factor required for activity of the Ah (dioxin) receptor. Science 1991;252:954-8.

24. Choi H, Kim SH, Chun YS, Cho YS, Park JW, Kim MS. in vivo hyperoxic preconditioning prevents myocardial infarction by expressing Bcl-2. Exp Biol Med 2006;231: 463-72.

25. Ohtsuka T, Zhou T. Bisindolylmaleimide VIII enhances DR5-mediated apoptosis through the MKK4/JNK/p38 kinase and the mitochondrial pathways. J Biol Chem 2002; 277:29294-303.

26. Wasem C, Arnold D, Saurer L, Corazza N, Jakob $S$, Herren $S$, et al. Sensitizing antigen-specific CD8+ T cells for accelerated suicide causes immune incompetence. J Clin Invest 2003;111:1191-9.

27. Pasgaard T, Stankevicius E, Jorgensen M, Ostergaard L, Simonsen U, Frobert 0. Hyperoxia reduces basal release of nitric oxide and contracts porcine coronary arteries. Acta Physiol 2007;191:285-96.

28. Kim J, Kim OS, Kim CS, Kim NH, Kim JS. Cytotoxic role of methylglyoxal in rat retinal pericytes: Involvement of a nuclear factor-kappaB and inducible nitric oxide synthase pathway. Chem Biol Interact 2010; 188:86-93. 